\title{
THERMAL IGNITION IN A REACTIVE SLAB WITH UNSYMMETRIC BOUNDARY TEMPERATURES
}

\author{
K. K. TAM ${ }^{1}$ and P. B. CHAPMAN ${ }^{2}$
}

(Received 13 April 1981; revised 8 March 1982)

\begin{abstract}
The problem of thermal ignition in a reactive slab with unsymmetric temperatures equal to 0 and $T$ is considered. Steady state upper and lower solutions are constructed. It is found that $T$ plays a critical role. Results similar to the case with symmetric boundary temperatures are expected when $T$ is small. When $T$ is sufficiently large, there is only one steady state upper or lower solution. The time dependent problem is then considered. Phenomena suggested by studying the upper and lower steady state solutions are confirmed.
\end{abstract}

\section{Introduction}

The stationary problem of thermal ignition in a reactive slab with unsymmetric boundary temperatures is a logical extension of the problem with symmetric boundary temperatures. As such, there have been a number of investigations on the problem, starting from the early work of Zel'dovich and Semenov (for references to early work, see Frank-Kamenetskii [1]), to the work of Shouman and Donaldson [2]. Using an exact solution method, Shouman and Donaldson demonstrated the existence of two types of steady state temperature profiles for a given set of boundary conditions. One solution describes the case of cooling at both surfaces and the other describes the case where one surface is heated and the second surface is cooled. The authors in [2] refer to both solutions as exhibiting thermal runaway. However, in the first case, the temperature has an interior

\footnotetext{
' Department of Mathematics, McGill University, Montreal, Quebec H3C 3G1, Canada.

${ }^{2}$ Department of Mathematics, The University of Western Australia, Nedlands, Western Australia 6009.

(c) Copyright Australian Mathematical Society 1983
} 
maximum, while in the second case, the temperature is monotonic with its maximum value given by the prescribed temperature at one wall. Thus, the two types of solutions are qualitatively different. We emphasize this difference by referring to the first type solution as having an interior maximum, and the second type as monotonic. Recently, Tam [3] has used a comparison theorem to construct upper and lower solutions for the problem with symmetric boundary temperatures, and obtained some results concerning the role of the initial temperature distribution in determining the steady state, in the event there are multiple steady state solutions. The role of the initial data was further studied in [4] where the time dependent problem was considered.

In this note, we use the same approach to deal with the problem with unsymmetric boundary temperatures. An interesting though perhaps not surprising result is that for $\alpha$ and $\delta$ in a certain domain, the type of solution that results depends on $T$. For the case of $T=0$, it is well-known that there is a critical value for the parameter $\delta$, which is dependent on $\alpha$, such that the steady state solution is super-critical if $\delta>\delta_{\mathrm{cr}}(\alpha)$, and sub-critical if $\delta<\delta_{\mathrm{cr}}(\alpha)$. In the present case, the critical value of $\delta$ depends on both $\alpha$ and $T$, and $\delta_{\mathrm{cr}}(\alpha, T)<\delta_{\mathrm{cr}}(\alpha)$. Further, numerical results suggest that $\delta_{\mathrm{cr}}(\alpha, T)$ is a decreasing function of $T$. Thus, a situation that is sub-critical when $T=0$ becomes super-critical when $T$ is sufficiently large.

In Section 2, we construct upper and lower solutions for the steady state. In Section 3, we examine the role of the boundary temperature and show that multiple upper and lower steady state solutions can exist only if $T$ is sufficiently small. In Section 4, we follow the approach used in [4] to rewrite the initial value problem as an integral equation, then use asymptotic considerations to obtain information about the influence of the initial and boundary data. Results similar to those for the case of $T=0$ are obtained. Further, consistent with the findings of Section 3, it is seen that for a given $\alpha$, there is a transitional value of $T(\alpha)$ above which there is no multiple steady state solution to the initial value problem. Thus, we have a new parameter in the phenomenon of "disappearance of criticality". The values of $T_{\text {tr }}(\alpha)$ for a few values of $\alpha$ are obtained for illustrative purposes.

The time dependent problem is formulated as the system

$$
\begin{gathered}
P \theta=\frac{\partial \theta}{\partial t}-\frac{\partial^{2} \theta}{\partial x^{2}}-\delta \exp \left(\frac{\alpha \theta}{\alpha+\theta}\right)=0, \quad t>0, \quad 0<x<1, \\
\theta(0, t)=T, \quad \theta(1, t)=0, \quad t>0 \\
\theta(x, 0)=h_{1}(x), \quad 0 \leqslant x \leqslant 1,
\end{gathered}
$$

where $\delta, \alpha$ and $T$ are positive parameters (see Frank-Kamenetskii [1]). 


\section{The steady state upper and lower solutions}

We seek an upper solution of the form

$$
U=(1-x)(b x+T(1+x)),
$$

where the parameter $b$ is to be determined. Since $d U / d x=b$ at $x=0$, it follows that when $b>0, U$ has a local maximum at $x_{m}=\frac{1}{2} b /(b+T)$ where $U\left(x_{m}\right)$ $=\frac{1}{4}(b+2 T)^{2} /(b+T)$. When $b<0$, the maximum value of $U$ in $0 \leqslant x \leqslant 1$ is $U(0)=T$. Here, we observe that it is sufficient to consider $b>-T$, as an upper solution must lie above the straight line joining $\theta=T$ at $x=0$ and $\theta=0$ at $x=1$. The value of $b$ is determined from the condition that $U$ be an upper solution, that is $P U \geqslant 0$. We have

$$
P U=2(b+T)-\delta \exp \left(\frac{\alpha U}{\alpha+U}\right) .
$$

To ensure $P U \geqslant 0$, we ask that

$$
2(b+T)-\delta \exp \left(\frac{\alpha U_{\max }}{\alpha+U_{\max }}\right)=0,
$$

since the exponential function $\exp \left(\frac{\alpha U}{\alpha+U}\right)$ is an increasing function of $U$. For $b>0, U_{\max }=U\left(x_{m}\right)$ and (4) becomes

$$
2 s=\delta \exp \left(\frac{\alpha(s+T)^{2}}{4 \alpha s+(s+T)^{2}}\right),
$$

where we have set $s=b+T$.

The exponential function equals $e^{\alpha}$ at $s=0$, is asymptotic to $e^{\alpha}$ as $s$ tends to infinity, and has a local minimum at $s=T$ with value $\exp (\alpha T /(\alpha+T))$. Thus, a solution for $b$ is obtained from (5) for $b>0$ and from

$$
2 s=\delta \exp \left(\frac{\alpha T}{\alpha+T}\right)
$$

for $0<s<T$ or $-T<b<0$. It can be readily verified that depending on the magnitude of the parameters, there may be one, two or three solutions. We shall examine the detailed situation in the next section.

We seek a lower solution of the form

$$
u=C \sin ^{2} \pi y,
$$

where $C=(a+2 T)^{2} / 4(a+T)>0$ and $y=((a+T) x+T) /(a+2 T)$. The parameter $a$ introduced here is to be determined, but $a>-T$. The interval $0 \leqslant x \leqslant 1$ becomes $0<T /(a+2 T) \leqslant y \leqslant 1$. For $u$ to be a lower solution, we must first ascertain that $\left.u(0) \equiv u\right|_{x=0} \leqslant T$. This can be verified by showing that $u(0)=T$ when $a=0, d u(0) / d a<0$, for $a>0$ and $d u(0) / d a>0$ for $a<0$; a 
straightforward, though somewhat cumbersome procedure. Next, we have $P u=$ $-\frac{1}{2} \pi^{2}(a+T) \cos 2 \pi y-\delta \exp \left(\left(\alpha C \sin ^{2} \pi y\right) /\left(\alpha+C \sin ^{2} \pi y\right)\right)$. For $0 \leqslant y \leqslant \frac{1}{4}$, and $\frac{3}{4} \leqslant y \leqslant 1$, we have $\cos 2 \pi y \geqslant 0$, so that $P u<0$. Further, we have $\cos 2 \pi y<0$ and $\sin ^{2} \pi y>\frac{1}{2}$ for $\frac{1}{4}<y<\frac{3}{4}$. Thus, $u$ will be a lower solution, that is $P u \leqslant 0$ if $a$ is determined from the equation

$$
\pi_{2}^{2}(a \mid T)=\delta \operatorname{e} \overline{\mathrm{p}}\left(\frac{\alpha C}{2 \alpha+C}\right),
$$

or

$$
\frac{\pi^{2}}{2} p=\delta \exp \left(\frac{\alpha(p+T)^{2}}{8 \alpha p+(p+T)^{2}}\right)
$$

where $p=a+T$. The exponential function on the right hand side of (7) equals $e^{\alpha}$ at $p=0$, is asymptotic to $e^{\alpha}$ as $p$ tends to infinity, and has a local minimum at $p=T$ with value $\exp (\alpha T /(2 \alpha+T))$. Again, depending on the magnitude of the parameters, (7) may have one, two or three solutions.

\section{The influence of the boundary temperature on the upper and lower solutions}

To investigate whether there is more than one upper solution of the form sought, we have to consider the pair of simultaneous equations consisting of (5) and the equation obtained by differentiating (5) with respect to $s$. For given $\alpha$ and $T$, if the simultaneous equations have two positive solutions, then one can use them to obtain two values for $\delta$, say $\delta_{2}(\alpha, T)$ and $\delta_{4}(\alpha, T)>\delta_{2}(\alpha, T)$, such that there will be three upper solutions for $\delta_{2}<\delta<\delta_{4}$, and two upper solutions for $\delta=\delta_{2}$ or $\delta=\delta_{4}$. This result is evident from a consideration of the logistic-shaped curve given by the right hand side of (5). Now, the pair of equations can be reduced by elimination to yield the quartic equation

$$
\begin{aligned}
s^{4}+4\left(T+2 \alpha-\alpha^{2}\right) s^{3} & +2\left(3 T^{2}+8 \alpha T+8 \alpha^{2}\right) s^{2} \\
+4 T^{2}\left(T+2 \alpha+\alpha^{2}\right) s+T^{4} & =0 .
\end{aligned}
$$

It is clear that if $T$ is sufficiently large, (8) has no positive real solution. The value of $T$ above which (8) has no real positive solution depends on $\alpha$, say $T_{L}(\alpha)$. Taking an increment of one unit in $T$, we have computed the following result on $T_{L}(\alpha)$ for a range of values of $\alpha$, and shown it in Table 1 .

TABLE 1 . The quantity $T_{L}(\alpha)$ as a function of $\alpha$

\begin{tabular}{|c|l|l|l|l|l|l|l|l|l|l|l|l|l|l|l|l|}
\hline$\alpha$ & 5 & 6 & 7 & 8 & 9 & 10 & 11 & 12 & 13 & 14 & 15 & 16 & 17 & 18 & 19 & 20 \\
\hline$T_{L}(\alpha)$ & 3 & 6 & 9 & 14 & 19 & 25 & 32 & 39 & 48 & 57 & 67 & 77 & 88 & 100 & 130 & 135 \\
\hline
\end{tabular}


From the above, we can draw the following conclusion: For a fixed $\alpha$ and $T>T_{L}(\alpha)$, there is a unique upper solution of the form sought, given either by the solution of (5) or (6). Which equation is to be used depends on the magnitude of $\delta$, with the demarkation value given by

$$
\delta_{L}(\alpha, T)=2 T \exp \left(-\frac{\alpha T}{\alpha+T}\right)
$$

If $\delta<\delta_{L}$, we have $d U /\left.d x\right|_{x=0}=b<0$ and since $U^{\prime \prime}<0$, we have $d U / d x<0$ for $0 \leqslant x \leqslant 1$. Thus, the upper solution will have negative slope in $0<x<1$, indicating that heat flows into the slab at $x=0$ and out at $x=1$. As for the actual solution, since $\partial^{2} \theta / \partial x^{2}<0$ for $0<x<1$ and $\partial \theta /\left.\partial x\right|_{x=0} \leqslant b<0, \theta$ must be monotonic decreasing for $0<x<1$ if $\delta<\delta_{L}$. If $\delta>\delta_{L}$, the upper solution will have an interior maximum, but this does not imply the solution itself must necessarily have an interior maximum also.

Entirely analogous considerations given to the lower solution yield similar results. The algebraic equation corresponding to (8) is

$$
\begin{aligned}
p^{4}+4\left(T+4 \alpha-2 \alpha^{2}\right) p^{3} & +2\left(3 T^{2}+16 \alpha T+32 \alpha^{2}\right) p^{2} \\
+ & 4 T^{2}\left(T+4 \alpha+2 \alpha^{2}\right) p+T^{4}=0 .
\end{aligned}
$$

The value of $T$ above which (10) has no real positive solution is tabulated as a function of $\alpha$ in Table 2.

TABLE 2. The quantity $T_{U}(\alpha)$ as a function of $\alpha$

\begin{tabular}{|l|l|l|l|l|l|l|l|l|l|l|l|l|l|l|l|l|}
\hline$\alpha$ & 5 & 6 & 7 & 8 & 9 & 10 & 11 & 12 & 13 & 14 & 15 & 16 & 17 & 18 & 19 & 20 \\
\hline$T_{U}$ & 5 & 11 & 18 & 27 & 38 & 50 & 63 & 78 & 95 & 113 & 133 & 154 & 177 & 201 & 227 & 254 \\
\hline
\end{tabular}

For fixed $\alpha$ and $T>T_{U}$, we have the demarkation value for $\delta$ corresponding to (9)

$$
\delta_{U}(\alpha, T)=\frac{\pi^{2}}{2} T \exp \left(-\frac{\alpha T}{2 \alpha+T}\right)
$$

If $\delta>\delta_{U}$, (7) has a unique solution with $p>T$. Thus, we have $d u /\left.d x\right|_{x=0}=$ $(\pi(p+T) / 4) \sin (2 \pi T / p+T)>0$ and $C=(p+T)^{2} / 4 p>T$, implying that the lower solution has an interior maximum greater than $T$. Hence the actual solution must also have an interior maximum greater than $T$ when $\delta>\delta_{U}$. When $T$ is small enough for both the upper and lower solutions to have multiple solutions, the roles of $\delta$ and the initial temperature are as in the case with symmetric boundary temperatures, and we expect similar results as in [4] to hold. 


\section{The time dependent problem and the influence of the initial and boundary data}

In the previous section, we have shown that the surface temperature $T$ plays a significant role in affecting the number of possible upper and lower solutions of the forms sought. This phenomenon is reminiscent of the roles played by $\alpha$ and $\delta$. To have more definitive iñformatioun ôn the influeñce of $T$ ôn the niumber of steady state solutions, we study the time dependent problem along the lines detailed in [4].

We reformulate (1) by writing $\theta(x, t)=T(1-x)+\psi(x, t)$ for $t>0$. Equation (1) then becomes

$$
\frac{\partial \psi}{\partial t}=\frac{\partial^{2} \psi}{2 x^{2}}+\delta Q(\psi), \quad t>0, \quad 0<x<1,
$$

where $Q(\psi)=\exp \{((\alpha T(1-x)+\alpha \psi) /(\alpha+T(1-x)+\psi))\}$.

The boundary and initial conditions are then

$$
\psi(0, t)=\psi(1, t)=0, \quad t>0
$$

and

$$
\begin{aligned}
\psi(x, 0) & =\theta(x, 0)-T(1-x) \\
& =h(x), \quad 0<x<1 .
\end{aligned}
$$

We then rewrite (11), (12) and (13) as an integral equation. From this point on, the treatment is exactly as in [4], and we shall simply quote the relevant results. Let $G(x, \xi, t)$ be the Green's function for the linear boundary value problem obtained from (11), (12) and (13) by omitting $Q(\psi)$. We have

$$
G(x, \xi, t)=2 \sum_{k=1}^{\infty} e^{-k^{2} \pi^{2} t} \sin k \pi x \sin k \pi \xi .
$$

The solution of (11), (12) and (13) can then be obtained from the integral equation

$$
\psi(x, t)=G(x, \xi, t) \cdot h(\xi)+\delta \int_{0}^{t} G(x, \xi, t-s) \cdot F(\psi(\xi, s)) d s,
$$

where $G(x, \xi, t) \cdot \phi(\xi) \equiv \int_{0}^{1} G(x, \xi, t) \phi(\xi) d \xi$. We define the iteration scheme

$$
\psi_{n+1}(x, t)=G(x, \xi, t) \cdot h(\xi)+\delta \int_{0}^{1} G(x, \xi, t-s) \cdot F\left(\psi_{n}(\xi, s)\right) d s,
$$

with

$$
\psi_{0}(\xi, s) \equiv h(\xi)
$$




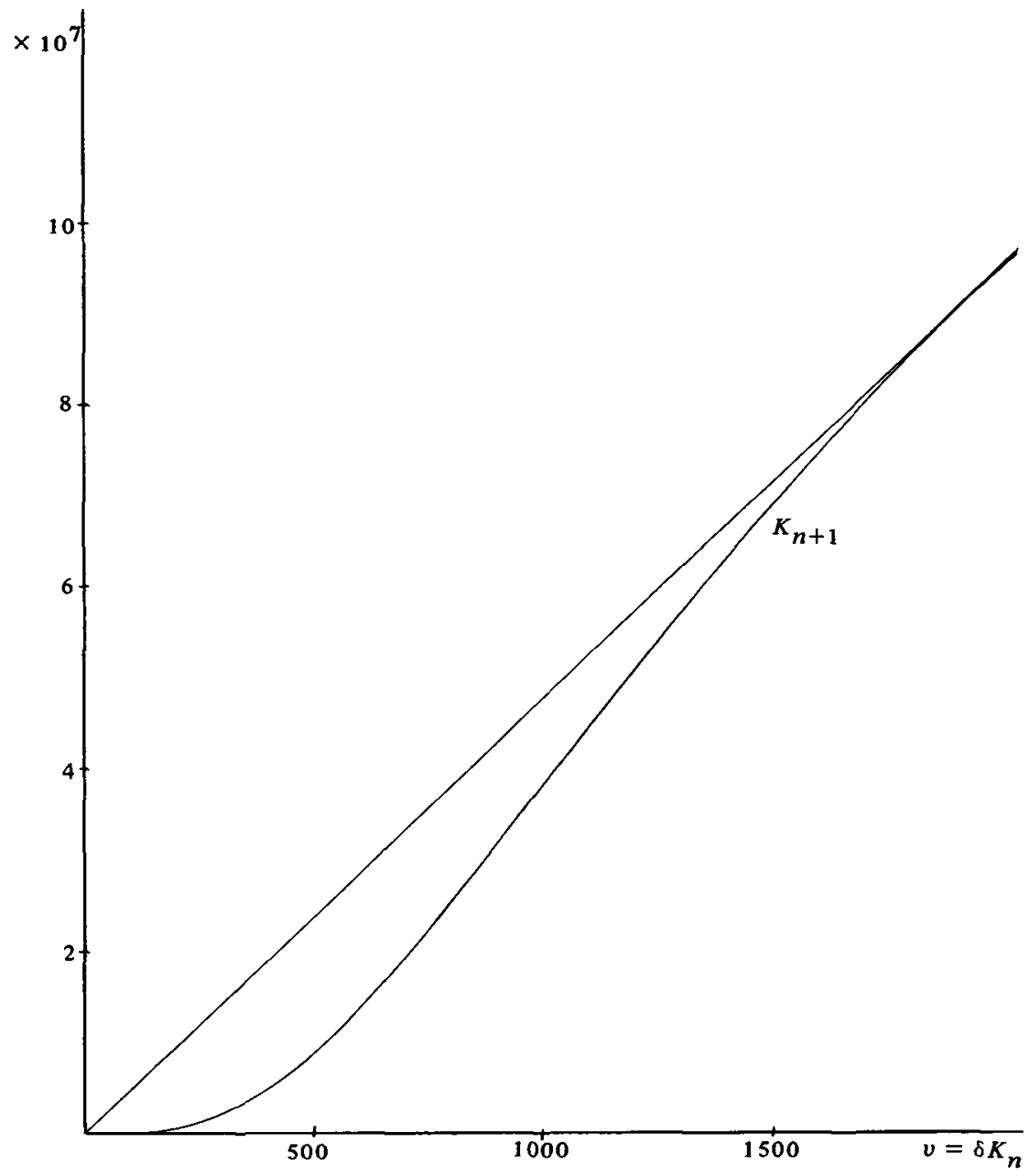

Figure 1. $K_{n+1}$ as a function of $v=\delta K_{n}$ for $\alpha=20, T=20$ with $\delta_{e}=2.10 \times 10^{-5}$ and $\delta_{\mathrm{cr}}=5.55 \times 10^{-3}$.

After some asymptotic analysis, it was found that in approximating $\psi_{n+1}$, we can write

$$
\psi_{n+1} \sim 2 \delta \sin \pi x \int_{\tau}^{t} e^{-\pi^{2}(t-s)} \sin \xi \cdot Q\left(\psi_{n}(\xi, s)\right) d s,
$$

where $\tau$ is sufficiently large.

Now suppose for $t>\tau$, we have $\sin \pi \xi \cdot Q\left(\psi_{n}(\xi, s) \sim K_{n}\right.$ for some $n$ where $K_{n}$ is independent of $s$. Then there exists $\tau_{n}>\tau$, such that for $t \gg \tau_{n}$, we have

$$
\psi_{n+1} \sim \frac{2 \delta K_{n}}{\pi^{2}} \sin \pi x
$$




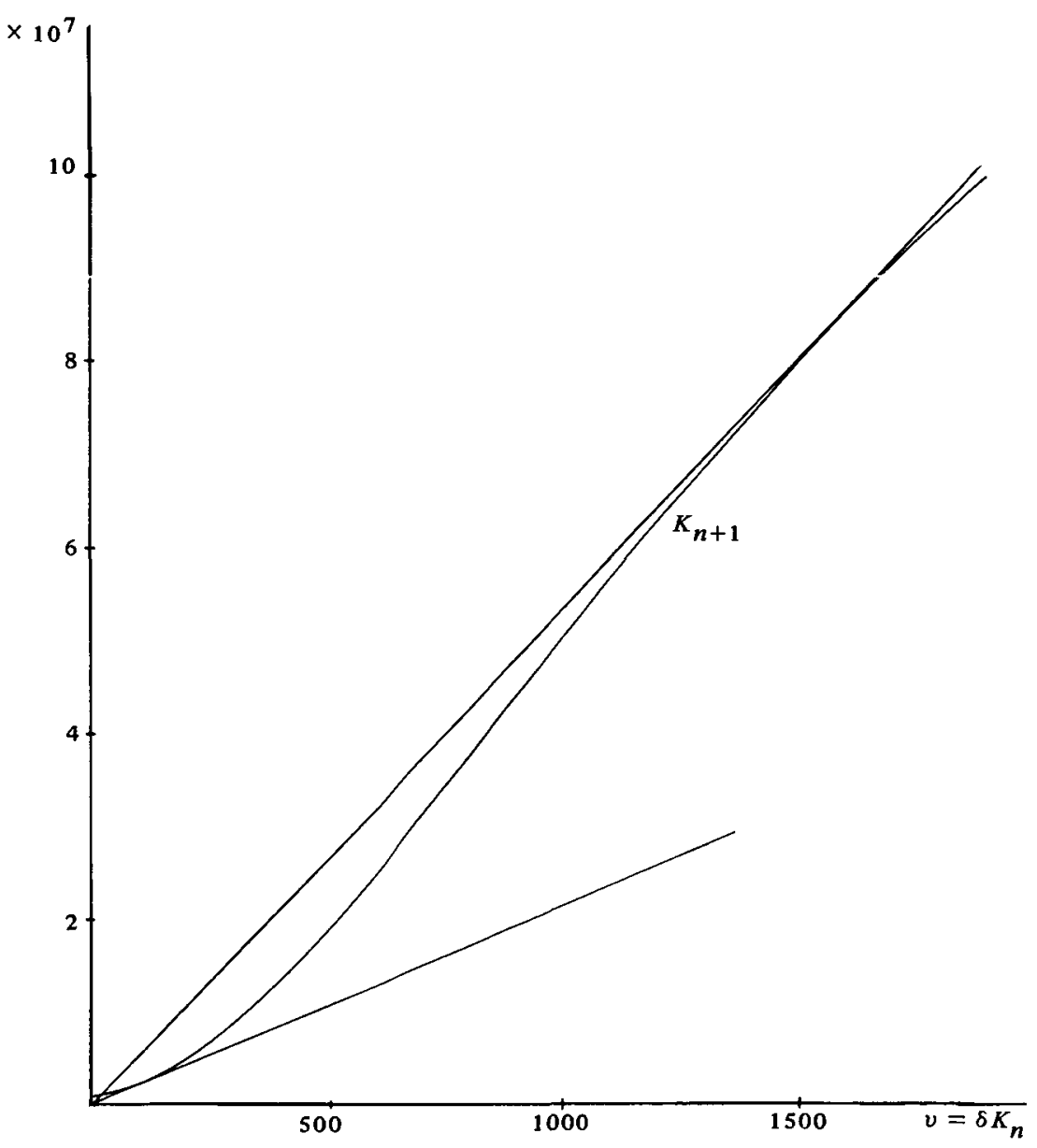

Figure 2. $K_{n+1}$ as a function of $v=\delta K_{n}$ for $\alpha=20, T=80$ with $\delta_{e}=1.88 \times 10^{-5}$ and $\delta_{\mathrm{cr}}=4.50 \times 10^{-5}$.

Using the above representation for $\psi_{n+1}$, we can proceed to consider $\sin \pi \xi$. $Q\left(\psi_{n+1}(\xi, s)\right)$. Supposing we have $\sin \pi \xi \cdot Q\left(\psi_{n+1}(\xi, s)\right) \sim K_{n+1}$, then, by repeating the above, we generate a sequence of numbers $K_{l}, i=n, n+1, \ldots$ We now compare $K_{n}$ with $K_{n+1}$. If, for a fixed $\delta$, we have $K_{n+1} \geqslant K_{n}$ for all $n$, the sequence $\left\{K_{t}\right\}$ is monotonic increasing. Conversely, if $K_{n+1} \leqslant K_{n}$ for all $n$, the sequence $\left\{K_{l}\right\}$ is monotonic decreasing. Since we know the solution $\psi$ is bounded, $\left\{K_{i}\right\}$ tends to a limit. If the limit $K_{\infty}$ is exponentially large, the solution of the initial value problem is super-critical. If $K_{\infty}$ remains small, the solution is sub-critical. 


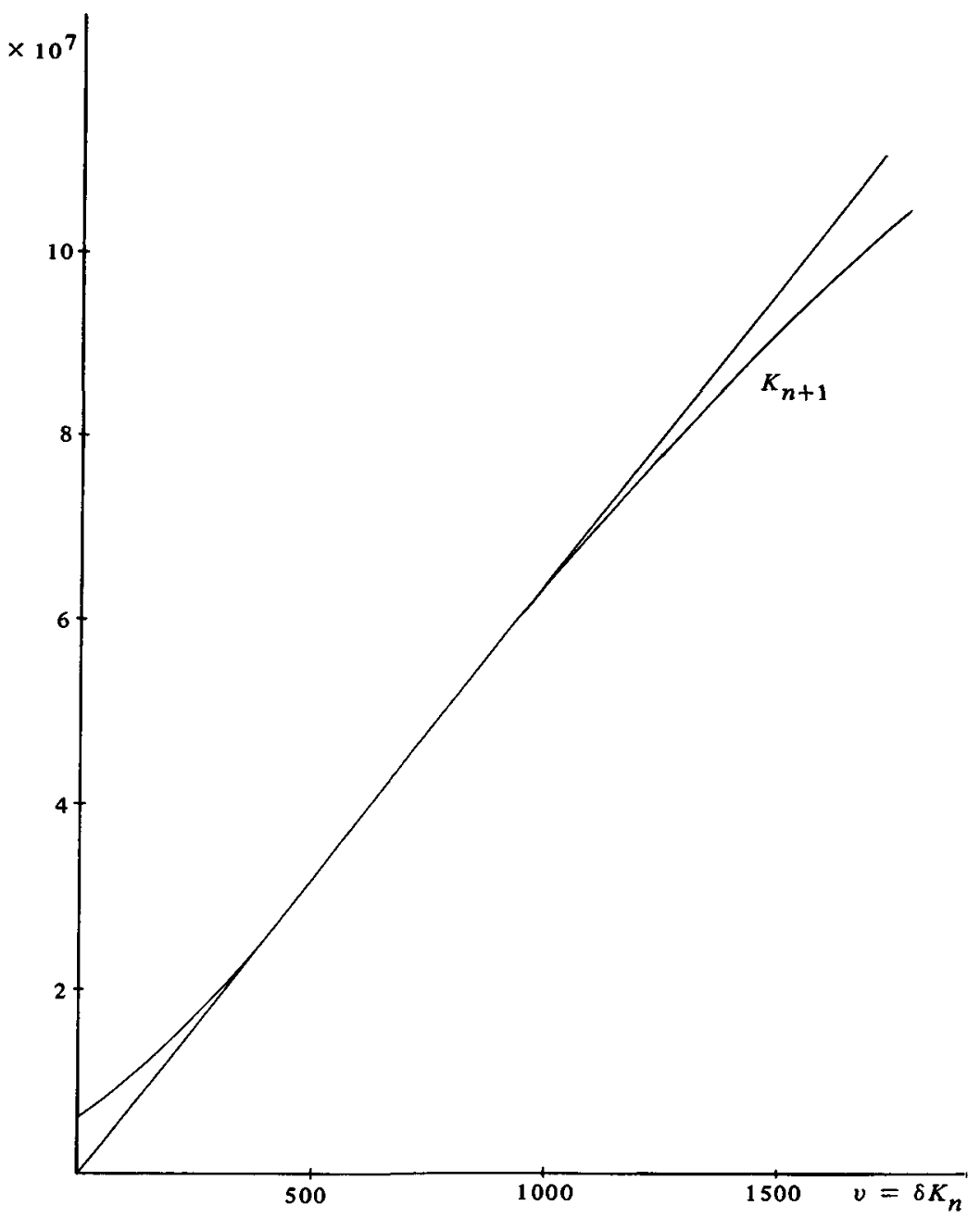

Figure 3. $K_{n+1}$ as a function of $v=\delta K_{n}$ for $\alpha=20, T=146$ with $\delta_{e}=\delta_{\mathrm{cr}}=1.57 \times 10^{-5}$.

To illustrate, we carry out some calculations for $K_{n+1}$ for $\alpha=20$, and various values for $T$. The results for $T=20,80$, and 146 are presented in Figures 1,2 and 3. For $T<146$, one can determine two values of $\delta$, say $\delta_{e}$ and $\delta_{\mathrm{cr}}$, such that for $\delta_{e}<\delta<\delta_{\mathrm{cr}}$, there are two stable steady states, and the initial data determines which one is reached. For $T \geqslant 146$, there is only one steady state, reached independently of the initial data. This situation is reminiscent of the "disappearance of criticality" in the case of $T=0$ when $\alpha$ approaches 4 . 


\section{Concluding remarks}

The initial and boundary value problem consisting of equations (1) to (3) offers a wealth of bifurcating phenomena, some of which are well-known. In this note, we have elucidated the role of the boundary temperature $T$. For $\alpha$ and $\delta$ in a certain range, the magnitude of $T$ determines whether there is one or two stable steady states for the problem. Viewed in a different way, a situation that is sub-critical for a given $T$ can become super-critical when $T$ is increased. In this sense, $T$ plays a "critical" role.

\section{Acknowledgements}

This work was initiated while the first author was a visitor at the University of Western Australia in 1979. The research was supported by the Natural Science and Engineering Research Council of Canada under Grant A-5228. The helpful comments of Dr. G. Wake of the Victoria University of Wellington are gratefully acknowledged.

\section{References}

[1] D. A. Frank-Kamenetskii, Diffuston and heat transfer in chemical kinetics (Plenum Press, 2nd Edition, 1969).

[2] A. R. Shouman and A. B. Donaldson, "The stationary problem of thermal ignition in a reactive slab with unsymmetric boundary temperatures", Combustion and Flame 24 (1975), 203-210.

[3] K. K. Tam, "Construction of upper and lower solutions for a problem in combustion theory", J. Math. Anal. Appl. 69 (1979), 131-145.

[4] K. K. Tam, "Initial data and criticality for a problem in combustion theory", J. Math. Anal. Appl. 77 (1980), 626-634. 